\title{
Protección contra la corrosión en agua de mar de la aleación AA5083 mediante tratamientos basados en sales lantánidas
}

\author{
A. Aballe $\left(^{(*)}\right.$, M. Bethencourt $\left(^{(*)}\right.$, F.J. Botana ${ }^{(*)}$, M. Marcos $^{(* *)}$, J. Pérez $^{(*)}$ y M.A. Rodríguez $\left(^{(*)}\right.$
}

\begin{abstract}
Resumen Se ha estudiado el poder protector de diferentes tratamientos superficiales obtenidos por inmersión total de muestras de la aleación AA5083 en disoluciones acuosas de $\mathrm{CeCl}_{3}, \mathrm{LaCl}_{3}$ y $\mathrm{SmCl}_{3}$. Su comportamiento frente a la corrosión en disoluciones de $\mathrm{NaCl}$ al 3,5\% se ha evaluado con ayuda de técnicas electroquímicas. Para ello, se ha determinado el grado de protección contra la corrosión uniforme y a la corrosión por picaduras, en términos del porcentaje de aumento de la resistencia a la nucleación de picaduras. A partir de estos resultados, se ha encontrado que las condiciones óptimas de protección se obtienen a partir de los 15 días de tratamiento en la disolución que contiene $\mathrm{CeCl}_{3}$, si bien mediante tratamientos de $48 \mathrm{~h}$ de inmersión se alcanzan grados de protección aceptables.
\end{abstract}

Palabras clave: Tratamientos superficiales no contaminantes. Aleaciones de aluminio. Elementos lantánidos.

\section{Protection against salt water of the AA5083 alloy by treatments based on lanthanides salts}

\begin{abstract}
AA5083 sheet samples have been pre-treated by full immersion in aqueous solutions of different lanthanide trichlorides salts, namely $\mathrm{CeCl}_{3}, \mathrm{LaCl}_{3}$ and $\mathrm{SmCl}_{3}$. The inhibition against uniform and pitting corrosion, in $3.5 \% \mathrm{NaCl}$ solutions, provided by such surface treatments has been estimated using electrochemical techniques. Protection against pitting has been quantified in terms of the percentage of increase observed for the pitting nucleation resistance. Among all the pretreatments tested, optimum performances have been observed for those carried out in $500 \mathrm{ppm} \mathrm{CeCl}_{3}$ aqueous solutions for fifteen days. However, acceptable protection degrees are observed even after $48 \mathrm{~h}$ in this solution.
\end{abstract}

Keywords: Environmental friendly surface pretreatments. Aluminium alloys. Lanthanide elements.

\section{INTRODUCCIÓN}

La legislación, en lo que se refiere al medio ambiente, resulta cada día más restrictiva en relación con aquellos procesos que implican la manipulación y vertido de sustancias con un elevado impacto ambiental. En ese sentido, uno de los sectores que se ha visto afectado por este tipo de res-

(*) Dpto. de Ciencia de los Materiales e Ingeniería Metalúrgica y Química Inorgánica. Univ. de Cádiz. Fac. de Ciencias del Mar. Aptdo 40. 11510-Puerto Real. (Cádiz, España).E-mail: javier.botana@uca.es

(**) Dpto. de Ingeniería Mecánica y Diseño Industrial. Univ. de Cádiz. Esc. Superior de Ingeniería. c/ Chile s/n. 11003Cádiz (España). tricciones es el relacionado con la industria de tratamientos de acabado superficial, especialmente aquellos que implican la utilización de compuestos de $\mathrm{Cr}(\mathrm{VI})$ (1 y 2). Por esta razón, desde hace algunos años se viene trabajando en la búsqueda de métodos alternativos a aquellos en los que se utilizan cromatos, que posean su misma eficacia anticorrosiva, pero que su impacto en el medio ambiente sea menor. Entre las distintas alternativas recogidas en la bibliografía se encuentra la basada en el empleo de compuestos de elementos lantánidos, tanto en lo que se refiere a su uso como inhibidores como en lo relativo a la formación de capas protectoras sobre distintas aleaciones metálicas (2-7), Estos compuestos se han utilizado con eficacia en 
la protección contra la corrosión de diversas aleaciones de aluminio de uso en la industria aeroespacial, como la AA2024, AA6061 ó AA7075 (8-10).

Desde hace algunos años, en nuestros laboratorios se viene trabajando en el estudio del poder inhibidor de diversas sales lantánidas frente a la corrosión en agua de mar de la aleación AA5083 (Al-Mg), especialmente utilizada en la construcción naval. En este trabajo se presentan los resultados correspondientes a la preparación y caracterización de las capas obtenidas, sobre la aleación AA5083, por inmersión en disoluciones que contenían cloruros lantánidos.

\section{PROCEDIMIENTO EXPERIMENTAL}

Los tratamientos superficiales se han realizado sobre muestras de la aleación AA5083 (Al-4,5\% $\mathrm{Mg}$ ) de $30 \times 25 \times 4 \mathrm{~mm}$. Para ello, se han utilizado disoluciones aireadas que contenían, respectivamente, $500 \mathrm{ppm}$ de $\mathrm{CeCl}_{3}, \mathrm{LaCl}_{3}$ y $\mathrm{SmCl}_{3}$, con y sin adición de $35 \mathrm{~g} / \mathrm{L}$ de $\mathrm{NaCl}$. Los tiempos de tratamiento oscilaron entre 1 y 30 días. Previamente a los tratamientos de inmersión, las muestras se pulieron sobre papel de $\mathrm{SiC}$ de 220 y 500 grits y, seguidamente, se enjuagaron con acetona y agua destilada.

Para evaluar el grado de protección de las capas depositadas se realizaron ensayos de polarización lineal, en disolución aireada de $\mathrm{NaCl}$ al 3,5\%, utilizando un potenciostato de Parc EG\&G, modelo VersaStat, y otro de Solartron, modelo SI1287.

Las muestras recubiertas se caracterizaron, asimismo, en un microscopio electrónico de barrido JEOL 820-SM equipado con un analizador de dispersión de energías AN-10000 de Link.

\section{RESULTADOS Y DISCUSIÓN}

En trabajos previos realizados en nuestros laboratorios, se ha podido comprobar que los cloruros lantánidos se comportan como inhibidores de la corrosión uniforme y por picaduras de la aleación AA5083 en disoluciones de $\mathrm{NaCl}$ (3 y 11-13). Al igual que sobre otras aleaciones de aluminio $(8,9 \mathrm{y}$ 14), estos compuestos actúan como inhibidores catódicos. Esta propiedad puede ser aprovechada para diseñar métodos de obtención de recubrimientos basados en el empleo de compuestos de elementos lantánidos (7-10).

En la figura 1 se representan las curvas de polarización lineal correspondientes a muestras tratadas durante períodos variables, de 1 a 30 días, en disoluciones aireadas de $\mathrm{NaCl}$ al 3,5\%, que contenían 500 ppm de $\mathrm{CeCl}_{3}$. En dicha figura, se ha incluido, como referencia, la curva correspondiente a una muestra

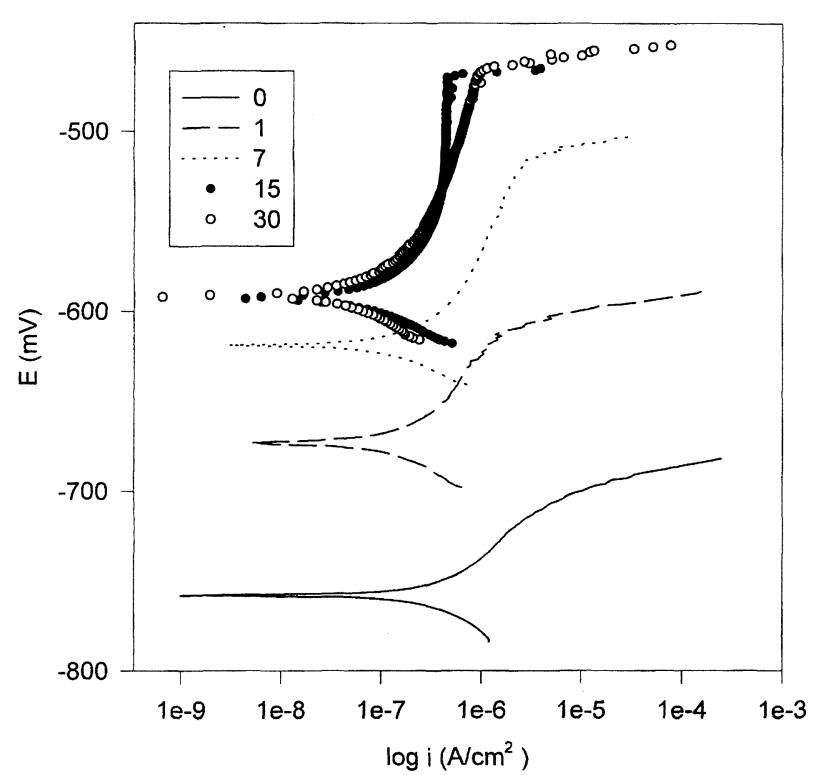

FIG. 1.- Curvas de polarización lineal de muestras de la aleación AA5083 tratadas por inmersión en disoluciones de $\mathrm{NaCI}$ al 3,5\% $\% 500$ ppm de $\mathrm{CeCl}_{3}$. Sobre la figura se indica la duración de los tratamientos en días. Medio agresivo: disolución de $\mathrm{NaCl}$ al 3,5\%.

FIG. 1.- Linear polarisation curves corresponding to AA5083 samples treated by full immersion in 3.5 $\% \mathrm{NaCl}+500 \mathrm{ppm} \mathrm{CeCl}_{3}$ solutions. Treatment times in days are indicated. Corrosive medium: $3.5 \% \mathrm{NaCl}$ solution.

de la aleación AA5083 sin tratar. Puede observarse que, desde el primer día de tratamiento, en las curvas de las muestras así tratadas, se produce un desplazamiento del potencial de corrosión hacia valores más nobles, respecto de la muestra sin tratar. Por otra parte, se detecta un descenso en los valores de las densidades de corriente de corrosión y, por tanto, de la velocidad de corrosión uniforme. Como puede comprobarse en la figura 2, al utilizar $\mathrm{LaCl}_{3} \mathrm{O}$ $\mathrm{SmCl}_{3}$, se obtienen comportamientos semejantes.

Haciendo uso de las curvas de polarización lineal, se ha determinado la velocidad de corrosión tras cada tratamiento. A partir de estos valores, se ha evaluado el grado de protección contra la corrosión uniforme, en términos del porcentaje de reducción, $P$, de la velocidad. En la figura 3 se muestra la evolución del parámetro $P$ con el tiempo de tratamiento para cada una de las disoluciones empleadas. En la citada figura puede observarse que al aumentar el tiempo de tratamiento aumenta el grado de protección contra la corrosión uniforme. Los resultados obtenidos ponen de manifiesto que, a partir del primer día, se supera el $50 \%$, y se alcanza el $99 \%$ a partir de los 15 días de exposición. Entre los distintos cloruros ensayados, el $\mathrm{CeCl}_{3}$ es el que conduce a porcentajes de protección más elevados. Por 


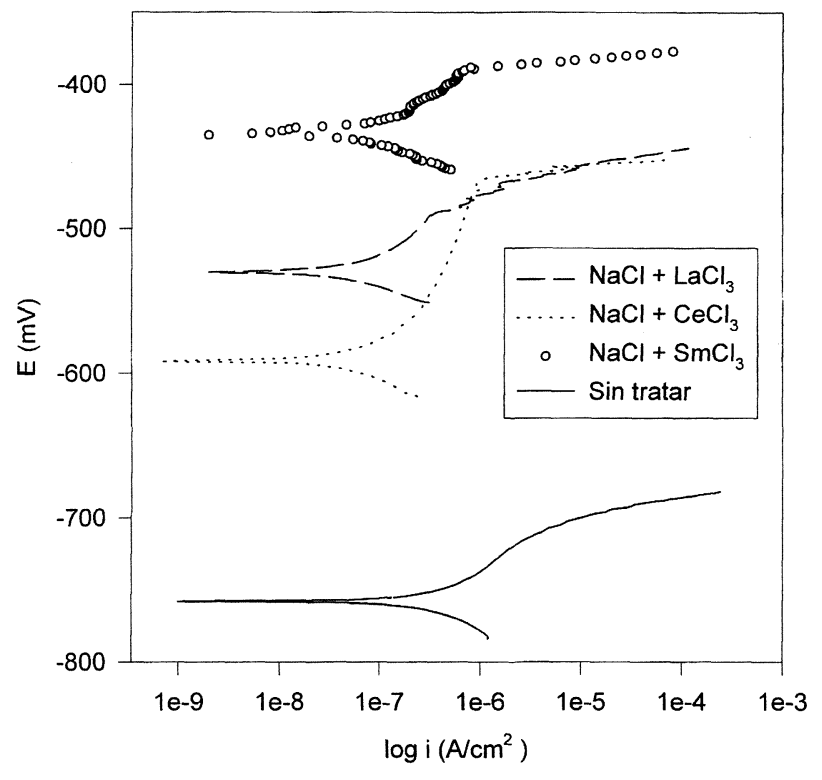

FIG. 2.- Curvas de polarización lineal de muestras de la aleación AA5083 tratadas por inmersión durante 30 días en las disoluciones que se indican.

Medio agresivo: disolución de $\mathrm{NaCl}$ al 3,5 \%.

FIG. 2.- Linear polarisation curves corresponding to AA5083 samples after a 30-days full immersion treatment in the referred solutions. Corrosive medium: $3.5 \% \mathrm{NaCl}$ solution.

último, cuando se comparan los valores de $P$ obtenidos para capas preparadas en disoluciones con y $\sin \mathrm{NaCl}$, se observa que se obtienen valores muy próximos. En resumen, los resultados incluidos en la figura 3, ponen de manifiesto, por una parte, que con los tratamientos propuestos se consigue reducir la velocidad de corrosión uniforme en niveles próximos al $100 \%$. Por otro lado, la presencia de $\mathrm{NaCl}$ en los baños de tratamiento parece tener poca influencia sobre el grado de protección contra la corrosión uniforme.

En las curvas de polarización lineal de las figuras 1 y 2 , es posible medir, además del potencial de corrosión, $E_{\text {corr }}$, el potencial de nucleación de picaduras, $E_{\text {np }}$. La resistencia a la nucleación de picaduras, $R_{\mathrm{np}}$, puede evaluarse a partir de la diferencia entre estos dos potenciales. El poder de protección frente a la nucleación de picaduras, suministrado por los diversos tratamientos, puede evaluarse en función del porcentaje de aumento, $P_{\mathrm{np}}$, de este parámetro (3 y 11). En la figura 4 se presenta la evolución de dicho parámetro en función del tiempo de tratamiento para las distintas disoluciones ensayadas. En esta figura puede observarse que, en los tratamientos realizados en disoluciones de $\mathrm{LaCl}_{3}$ ó $\mathrm{SmCl}_{3}, P_{\mathrm{np}}$ alcanza su valor máximo en torno al séptimo día de tratamiento. En el caso particular de las muestras tratadas en disoluciones

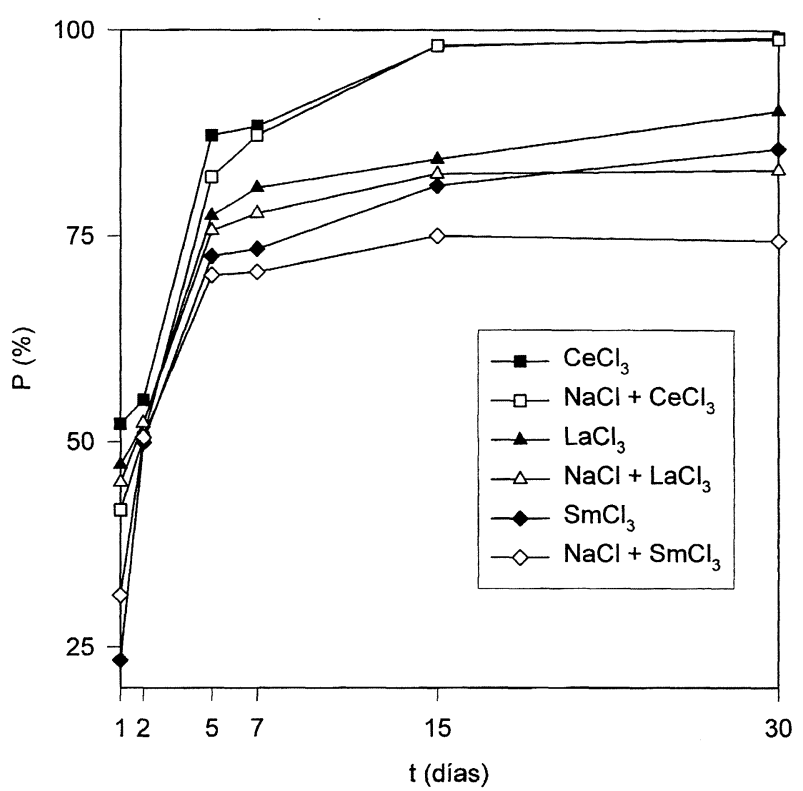

Fig. 3.- Porcentaje de protección frente a la corrosión uniforme en función del tiempo de tratamiento en las disoluciones que se indican. Medio agresivo: disolución de $\mathrm{NaCl}$ al 3,5\%.

FIG. 3.- Protection degree against uniform corrosion as a function of pretreatment time in the referred solutions. Corrosive medium: $3.5 \% \mathrm{NaCl}$ solution.

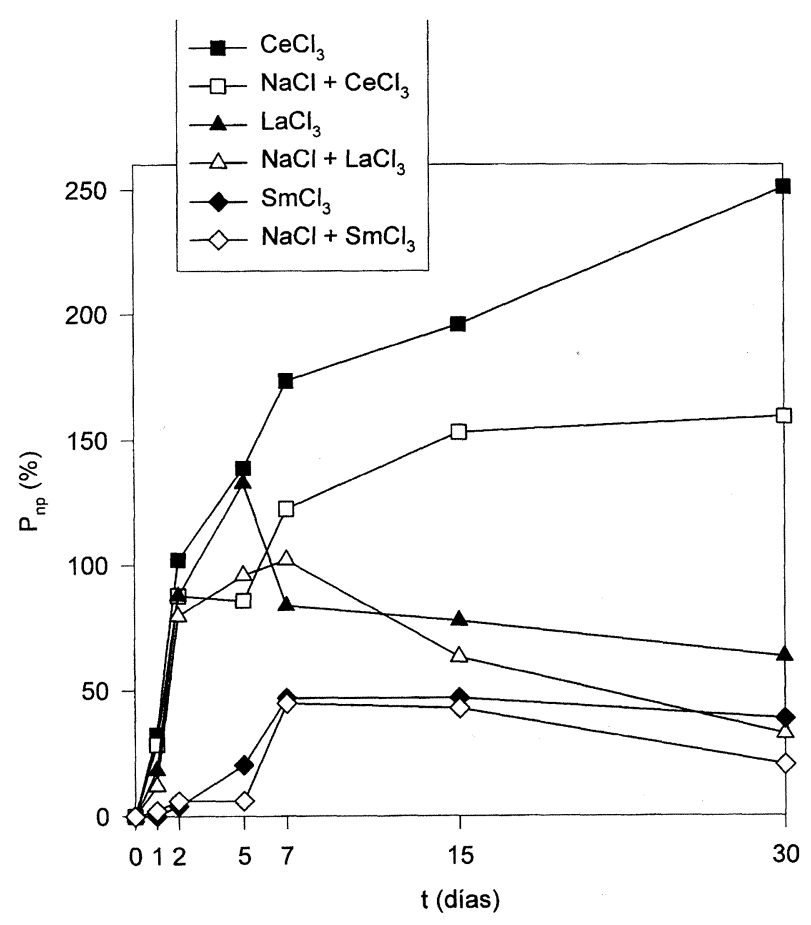

FIG. 4.- Porcentaje de protección frente a la corrosión por picaduras en función del tiempo de tratamiento en las disoluciones que se indican. Medio agresivo: disolución de $\mathrm{NaCl}$ al 3,5\%.

FIG. 4.- Protection degree against pitting corrosion as a function of pretreatment time in the referred solutions. Corrosive medium: $3.5 \% \mathrm{NaCl}$ solution. 
de $\mathrm{CeCl}_{3}$, el valor de $P_{\text {np }}$ aumenta continuamente. De hecho, son los tratamientos en $\mathrm{CeCl}_{3}$ los que conducen a mejores resultados, llegándose a superar el $200 \%$ a partir de los 15 días.

En la figura 4 puede observarse que, a diferencia de lo que ocurría con el porcentaje de protección contra la corrosión uniforme, existen diferencias de hasta un $100 \%$ entre los tratamientos realizados en disoluciones de $\mathrm{CeCl}_{3}$ con y sin $\mathrm{NaCl}$. En consecuencia, mientras que la presencia de $\mathrm{NaCl}$ en los baños de tratamiento no afecta al comportamiento de las muestras frente a la corrosión uniforme, sí
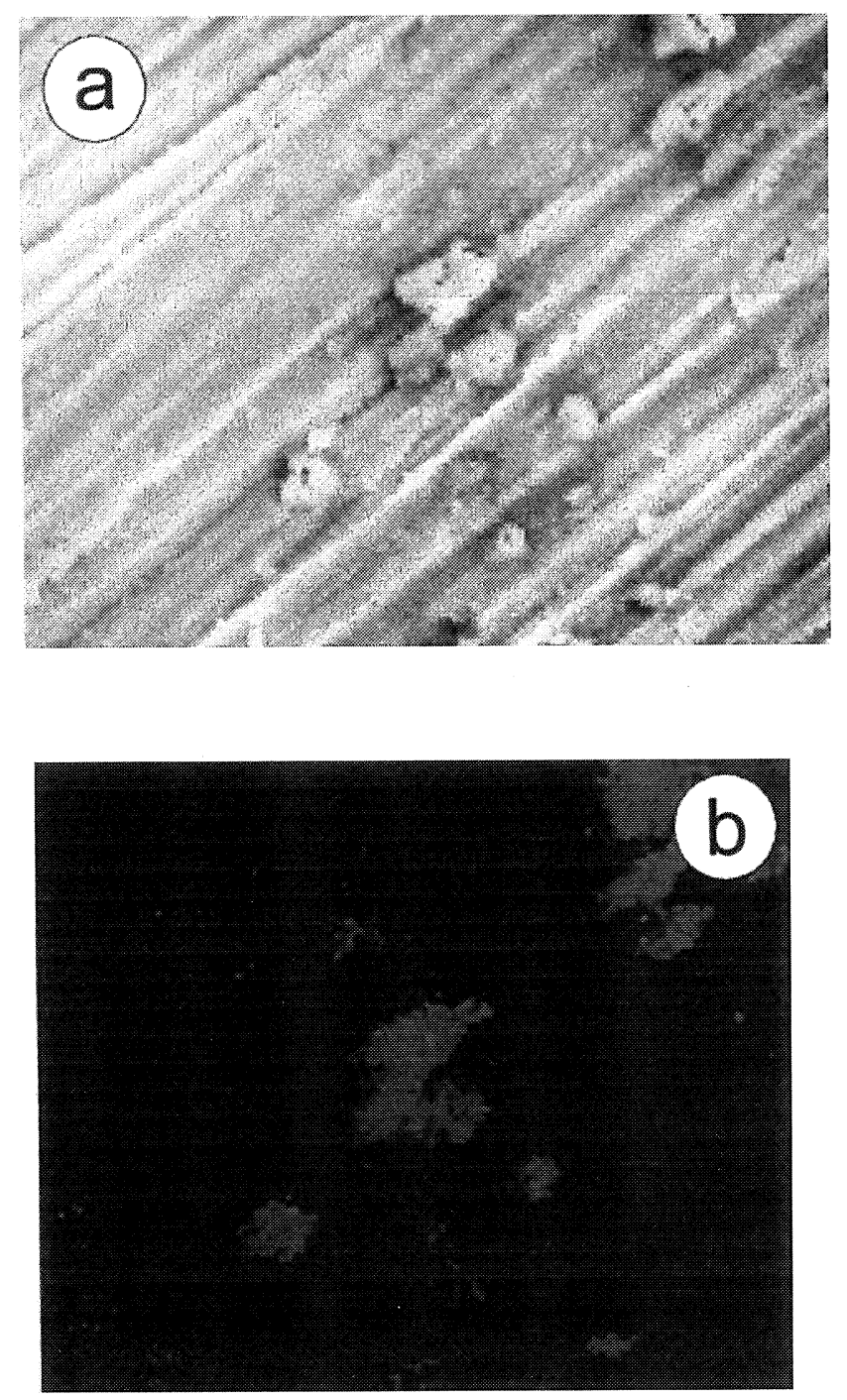

determina su comportamiento frente a la corrosión por picaduras.

De acuerdo con los resultados anteriores, los tratamientos que proporcionan una mayor mejora en el comportamiento general de la aleación son los realizados en disoluciones acuosas de $\mathrm{CeCl}_{3}$. Para determinar la naturaleza de las modificaciones superficiales que ocasionan dichas mejoras, las muestras tratadas fueron estudiadas mediante microscopía electrónica de barrido (MEB) y espectroscopía de dispersión de energías (EDS). La figura $5 a$ contiene una imagen MEB correspondiente a
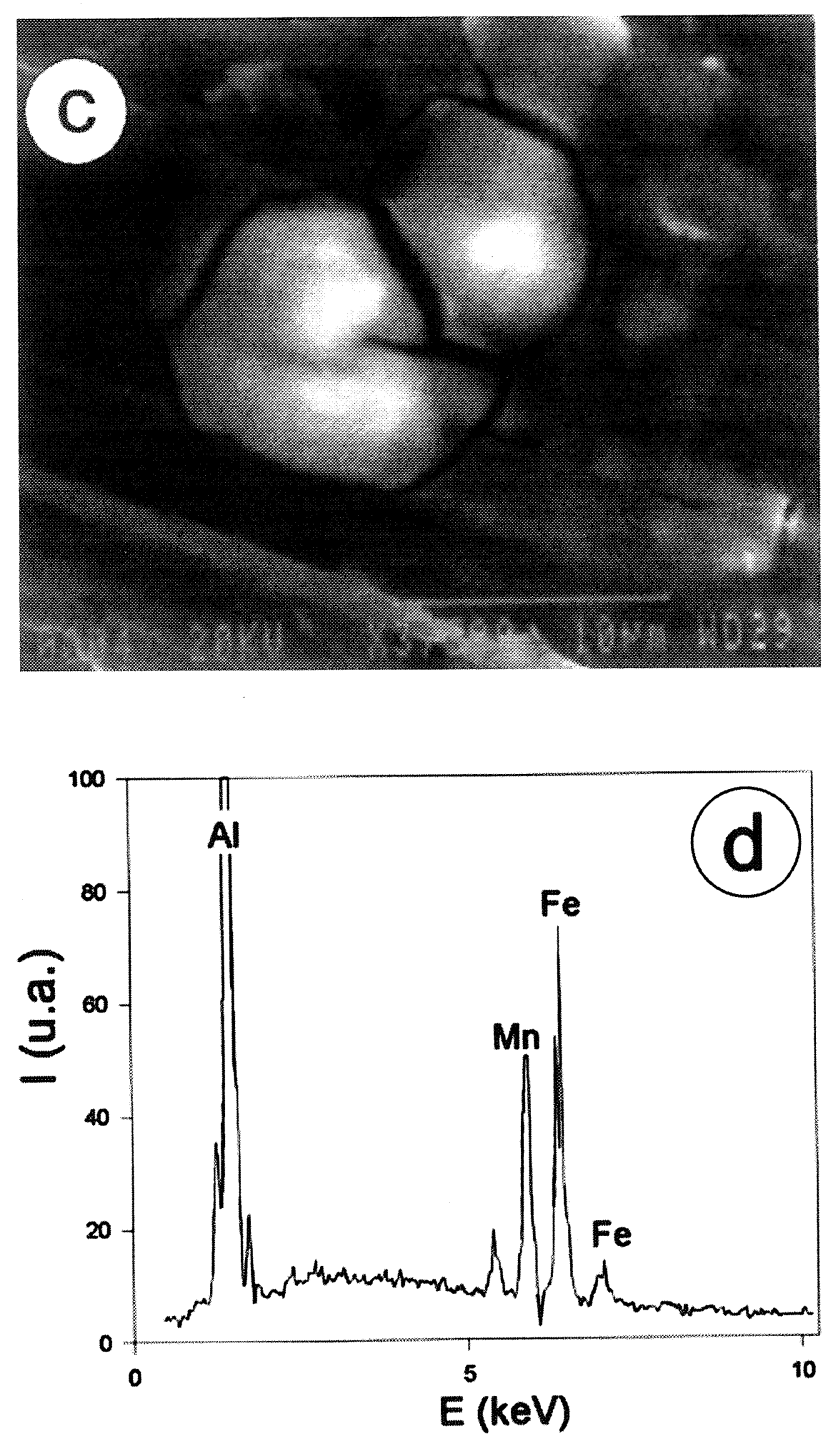

FIG. 5.- a) Imagen MEB de una muestra tratada durante 2 días en disolución de $500 \mathrm{ppm} \mathrm{de} \mathrm{CeCl}_{3} . \times 500 . b$ ) Mapas de distribución elemental de aluminio (oscuro) y cerio (claro) registrados en la imagen $a$ ). $c$ ) Imagen MEB de una muestra tratada durante $2 \mathrm{~h}$. Obsérvese la presencia de una isla de cerio. $\times 3000$. d) Espectro EDS adquirido en la zona de la muestra sobre la que se encuentran los depósitos de cerio.

FIG. 5.- a) SEM image corresponding to a sample treated during 2 days in a $500 \mathrm{ppm} \mathrm{CeCl}_{3}$ solution. $500 \times$;

b) Elemental distribution maps of aluminium (dark) and cerium (light) recorded in the region shown in a);

c) SEM image corresponding to a sample treated during $2 \mathrm{~h}$. A Ce-rich island can be observed. $3000 \times$. d) EDS spectrum acquired in the regions of the alloy underlying the cerium-containing islands. 
una muestra tratada durante $48 \mathrm{~h}$ en disolución aireada de $500 \mathrm{ppm} \mathrm{de} \mathrm{CeCl}_{3}$. En dicha imagen, puede apreciarse que, tras este tratamiento, la superficie de la muestra queda recubierta con una capa. El análisis mediante espectroscopía de dispersión de energías (Fig. $5 b$ ) ha permitido comprobar, en primer lugar, que dicha capa contiene, como elementos mayoritarios, aluminio y cerio, si bien la distribución de ambos elementos no es uniforme. De acuerdo con la información obtenida mediante EDS, el cerio se acumula en forma de islas (Fig. $5 c$ ), que se distribuyen irregularmente por la superficie. El espectro EDS representado en la figura $5 d$ fue registrado en las zonas del metal sobre el que crecen las islas de cerio. Dicho espectro pone de manifiesto que los depósitos de cerio se forman sobre los precipitados de $\mathrm{Al}_{6}(\mathrm{MnFe})$ existente en la aleación (15). Estos precipitados tienen carácter catódico respecto a la matriz de aluminio y actúan como núcleos en torno a los cuales tiene lugar la nucleación y propagación de las picaduras (15 y 16). De esa forma, en los primeros instantes del proceso de corrosión se generan grupos $\mathrm{OH}^{-}$sobre las zonas catódicas de la aleación, es decir, sobre los precipitados de ( $\mathrm{Al}, \mathrm{Mn}, \mathrm{Fe})$. Los iones $\mathrm{Ce}^{3+}$ existentes en la disolución reaccionarían con los grupos hidroxilos dando lugar a la formación de las islas ricas en cerio observadas en la figura 5. Estos resultados están de acuerdo con el mecanismo de inhibición propuesto en (14) para aleaciones de Al$\mathrm{Cu}$. La formación de estas islas traería consigo el bloqueo de las zonas catódicas del material, provocando, por tanto, un descenso en la velocidad de corrosión. Dado que la nucleación de las picaduras tiene lugar en las fronteras de dichos precipitados, la disminución de la actividad sobre los mismos trae consigo la inhibición del proceso de corrosión por picaduras.

\section{Agradecimiento}

Este trabajo ha sido financiado por la Comisión Interministerial de Ciencia y Tecnología (CICYT), Proyectos MAT97-1075-C03-01 y MAR95-2011.

\section{REFERENCIAS}

(1) Holmes, J. Metal Finish. 87 (11), 1989: 65.

(2) CoHEn, S.M. Corrosion, 51 (1), 1995: 71.

(3) Aballe, A., Bethencourt, M., Botana, F.J., Marcos, M., Pérez, J. y Rodríguez, M.A. Rev. Metal., 33 (6), 1997: 363.

(4) Bernal, S., Botana, F.J., Calvino, J.J., Cauqui, M.A., Marcos, M., Pérez, J.A. y Vidal, H. J. Alloys Comp., 225, 1995: 638 .

(5) Bernal, S., Botana, F.J., Calvino, J.J., Cauqui, M.A., Marcos, M. y Pérez, J.A. Proc. 5th Elec. Meth. Corr. Res., EMCR'94, Lisboa (Portugal). 1994. P-I1.

(6) SEON, F.M. J. Less Comm. Met., 148, 1989: 73.

(7) Hinton, B.R.W., Traten, P.N., Wilson, L. y Ryan, N.E. Proc. 28th Aust. Corr. Conf., Perth (Australia). 1988.

(8) Hinton, B.R.W., Hughes, A., Taylor, R., Henderson, M., Nelson, K. y Wilson, L. Proc. 13 th Int. Corr. Conf., Melbourne (Australia). 1996.

(9) Lin, S., Sinih, H. y Mansfeld, F. Corr. Sci., 33 (9), 1992: 1.331 .

(10) Wilson, L. y Hinton, B.R.W. Aust. Pat. P10649. 1987.

(11) Bethencourt, M., Botana, F.J., Cauqui, M.A., Marcos, M., RodríGuez-Chacon, M.A. y RodríGuezIZQUIERDO, J.M. J. Alloys Comp., 250, 1997: 455.

(12) Aballe, A., Bethencourt, M., Botana, F.J., Marcos, M., PÉrez, J. y Rodríguez, M.A. 6 ${ }^{\text {th }}$ Elec. Meth. Corr. Res., EMCR'97, Trento (Italia). 1997. P-A49.

(13) Aballe, A., Bethencourt, M., Botana, F.J., Marcos, M., Pérez, J. y Rodríguez, M.A. Mater. Sci. Forum. (En prensa).

(14) Aldykewicz, A.J., Isaacs, H.S. y Davenport, A.J. J. Electrochem. Soc., 143 (1), 1996: 147.

(15) Bethencourt, M., Botana, F.J., Marcos, M., Pérez, J. y Rodríguez, M.A. Proc. V Reunión Nacional de Materiales, Cádiz (España). 1996.

(16) Bethencourt, M., Botana, F.J., Calvino, J.J., Marcos, M., Pérez, J. y Rodríguez-Chacón, M.A. Mater. Sci. Forum. (En prensa). 